to $4 \%$. Success stories document the increased quality of life and ability to cope with stigma and discrimination.

Conclusions People living with HIV can be engaged in effective outreach when they function as spokes from the hub of a community care centre. Community outreach complements facility based clinical care and a comprehensive approach that includes both a bio-medical and social focus can improve quality of life and minimise death.

\section{P5-S6.25 INTEGRATING HIV/AIDS, FAMILY PLANNING, AND REPRODUCTIVE HEALTH SERVICE INTO NIGERIAN COMMUNITIES}

doi:10.1136/sextrans-2011-050108.581

C I Traffina. Institue of Human Virology of Nigeria, Abuja, Nigeria

Background In response to the rapid and complex HIV/AIDS epidemic, EPIC/DOD- Nigeria expanded its focus on family planning and reproductive health (FP/RH) to integrate HIV/AIDS prevention and care activities in 2008. Approaches include the establishment of work place programmes. Whereby peer promoters are selected from among the personnel. The peer promoters undergo a 2-week training that enables them to provide FP/RH and HIV/ AIDS- related information and services to their co-workers.

Methods The objective of the assessment was to identify the benefits of the workplace Programme as perceived by workplace organisations, peer promoters, and workers. The assessment was designed to obtain qualitative information through focus group discussions and reviews of client registration books.

Results Peer promoters educate workers during tea and lunch breaks. They also provide pills and condoms and refer to nearby clinics for injectables and other services. From 2008 to 2009, they served 2215 new family planning users in two sites mainly in the medical centers of Mogadishu cantonment- Asokoro - Abuja. Uptake of condoms is high-for dual protection against unintended pregnancy and STI/HIV/AIDS. Condoms are also available in workplace restrooms for soldiers and their families, especially at the Defence Health Club (DHC). Peer promoters are more than satisfied to provide this service, and say that since their involvement in the project, they know more about HIV/AIDS and other health issues. The management also acknowledges benefits of the Programme, both financially and in terms of workers health.

Conclusion Many people working in military units and establishments in Nigeria have reproductive health and HIV/AIDS needs. Due to the characteristics and timing of their work, they have limited access to needed FP/RH and HIV/AIDS information and services. Workplace and Programme benefit managers, peer promoters and workers and others they should be expanded in other barracks and defence locations across the nation.

\section{P5-S6.26 PREVENTION WITH POSITIVES IN NIGERIA: WHAT HAVE WE LEARNT?}

doi:10.1136/sextrans-2011-050108.582

\section{I Traffina. Institue of Human Virology of Nigeria, Abuja, Nigeria}

Background The introduction of care and treatment at the service delivery points at the community and the clinical settings- hospital facilities for prevention with the positives have giving the HIV/ AIDS, STIs, TB management high quality significant improvement in the first 8 months of implementation in Nigeria.
Methods Association of positive youth in Nigeria is a national nongovernmental organisation in Nigeria, involved in HIV prevention, care and support. APYIN started implementing HIV prevention strategies in 2009 with a focus on involving HIV - positive people in prevention. The strategies included; counselling on prevention of HIV to positive persons this involved discussion of behavioural intervention in medical and counselling visits. Promoting the use and provision of condoms to sexually active HIV-positive individuals, promoting adherence to antiretroviral drugs, by counselling, use of pills boxes, and medicine companions. Encouraging HIVpositive persons to disclose HIV status to sex partners either by self or counsellor mediated during home visits or at the nearest opportunity. Home base management, HIV counselling and testing targeting partners and children born to all people living with HIV/ AIDS.

Results Most of the persons living with HIV realise that they have a role to play in prevention of HIV. There is $8.5 \%$ increase in the uptake of condoms by HIV -positive person between the year 2008 and 2009. Antiretroviral therapy adherence levels of $92 \%$ of the clients on ART with adherence $>95 \%$. In the same period there is an increase of $50 \%$ of HIV -positive individuals disclosing HIV status to partners. Community awareness of HIV has increased in the area serviced by the organisation.

Conclusion Increase collaboration between the clinical and community base interventions. Regular monitoring of the community response for improvement. The capacity building of for community staff. HIV-positive persons are very important partners in HIV prevention. Therefore their involvement should be prioritised.

\section{P5-S6.27 NEW AVENUES TO INCREASE QUALITY OF STI-CARE IN GENERAL PRACTICE}

doi:10.1136/sextrans-2011-050108.583

J E A M van Bergen. Soa Aids Nederland Amsterdam, Netherlands

Background The role of primary care in STI-control has long been neglected. Although national STI surveillance often thrives on data of STI-Clinics, recent research in the Netherlands shows that approximately $70 \%$ of all STI-related consultations are in General Practice (GP). More attention for sexual health in primary care is warranted.

Methods In 2004 the Dutch College for Family Physicians (NHG) added a guideline about the "STI consultation" to their list of GP certified "standards of care". The guideline describes care for patients with complaints of STI, for patients with questions about STI (test request), and also describes pro-active testing policies for persons visiting the health centre for a not-STI related reason. The guideline is currently being updated. Implementation of the guideline is facilitated by regional "expert" GP, united in the GP-advisory group on STD, HIV and Sexuality (the Expert group Sexual Health (SeksHAG). Such Expert Groups within the GP institutional body are relatively new and also exist, for example, diabetes and Asthma/ COPD.

Results Annually approximately 1500 GP receive a continuous education session on STI facilitated by the GP expert in their region. Previous research showed moderate impact on testing habits and case detection. Qualitative interviews describe insight in personal barriers as a positive gain in training sessions. Prescribing habits of GP for Gonococcal infections are legging behind, half of GP prescribing ciprofloxacin, for which resistance is well above $40 \%$.

Discussion A substantial but often hidden proportion of STI consultations take place in general practice. More interventions on STI-care in GP are recommended. An expert group within the national GP body can enhance attention for quality of care. The new 